\title{
Polysèmes
}

Revue d'études intertextuelles et intermédiales

\section{Vita Sackville-West en Perse : les fruits du voyage, de la correspondance aux poèmes}

Isabelle Gadoin

\section{(2) OpenEdition}

\section{Journals}

Édition électronique

URL : http://journals.openedition.org/polysemes/674

DOI : 10.4000/polysemes.674

ISSN : 2496-4212

Éditeur

SAIT

Édition imprimée

Date de publication : 1 janvier 2012

Pagination : 129-153

ISSN : 0999-4203

\section{Référence électronique}

Isabelle Gadoin, «Vita Sackville-West en Perse : les fruits du voyage, de la correspondance aux poèmes », Polysèmes [En ligne], 12 | 2012, mis en ligne le 01 mars 2015, consulté le 30 avril 2019 URL : http://journals.openedition.org/polysemes/674; DOI : 10.4000/polysemes.674

Ce document a été généré automatiquement le 30 avril 2019

Polysèmes 


\title{
Vita Sackville-West en Perse : les fruits du voyage, de la correspondance aux poèmes
}

\author{
Isabelle Gadoin
}

«Je hais les voyages et les explorateurs " posait perfidement Claude Lévi-Strauss à l'incipit de ce qui pourrait pourtant être lu comme récit de voyage, Tristes Tropiques ${ }^{1}$. Or, comme en anticipation de cette formule lapidaire, Vita Sackville-West avait elle aussi persiflé : «there is no greater bore than the travel bore $»^{2}$. Les deux auteurs s'accordent dans le rejet du voyage en tant que tel, comme découverte exotique ou échappée romantique; et pourtant tous deux s'accordent à faire le récit de ces voyages, comme si la narration de l'expérience vécue permettait de dépasser, mais aussi de justifier, de racheter, voire de transcender le voyage. Vita Sackville-West semble ne pas avoir choisi de se rendre en Perse : elle se contenta d'y suivre son mari, le diplomate Harold Nicolson. Né à Téhéran en 1886, alors que son père Arthur Nicolson y représentait le gouvernement britannique, Nicolson fut à son tour posté à la Légation Britannique de Téhéran en novembre 1925. De ces voyages qui semblent en partie contraints, Vita Sackville-West tira néanmoins deux récits inspirés, Passenger to Teheran, publié en 1926, puis Twelve Days: An Account of a Journey in the Bakhtiari Mountains of South-Western Persia, en 1928. De ce rapport ambigu qu'entretiennent l'expérience du voyage et la production du récit, elle s'explique dans ses mots d'ouverture, ou préambule, «terme dont l'étymologie rappelle précisément l'idée du voyage $»^{3}$, de la déambulation. Ces deux moments liminaires des récits de voyage ne sont pas à proprement parler "hors-texte", car ils constituent à chaque fois le premier chapitre du récit, et pourtant ils s'en distinguent par leur caractère réflexif et polémique, qui leur confère véritablement la fonction de préface, "lieu d'une concentration, d'une condensation des enjeux, de la diversité et de la complexité de l'écriture voyageuse $»^{4}$. L'incipit de Passenger to Teheran ouvre le texte sur l'idée de l'impossibilité à narrer proprement le voyage - affirmation où l'on reconnaît le mythe de l'« impossible à dire », le topos des limites de la langue par rapport à un référent toujours insaisissable en son immédiateté et sa totalité. Ce cliché est évidemment l'une des autocomplaisances du voyageur (qui aurait vu, lors d'une expérience incomparable et 
inénarrable, ce que nul autre ne pourra même imaginer). Mais il se fonde aussi sur un autre cliché, celui de la totale extériorité des perceptions sensorielles au langage :

We look; and there is the image in its entirety, three-dimensional, instantaneous. Language follows, a tortoise competing with the velocity of light; and after five pages of print succeeds in reproducing but a fraction of the registered vision. [...] The most - but what a most! - that language can hope to achieve is suggestion; for the art of words is not an exact science. (PT 27)

2 La mystique en filigrane ici, c'est encore et toujours celle de la grandiose primauté de la chose et de la fatale et désespérante secondarité du mot, la mystique d'un langage postlapsarien. Le voyageur-conteur se verrait inéluctablement confisquer son propre voyage, en vertu de cette croyance à un référent «brut », posé dans un calme déjà-là, dans une relation d'absolue étrangeté à tout regardeur; un référent lointain, insaisissable, et forcément faussé par toute mise en mots. Or ce topos n'a pas même le temps d'être posé qu'il se voit déjà contesté, sans même que la préfacière s'en rende tout à fait compte, par la constatation fataliste :

Thought is impossible without words, and the process of thought appears to us as a desirable exercise; but how are we to know the relation of thought to the world of fact? whether any true relation at all, or merely the conventionalised, stylised relation such as is born by art, that extraordinary phenomenon, that supreme paradox of conveying truth through various conventions of falsity? (PT 28)

En s'empêtrant malgré elle dans son propre argument, Vita Sackville-West dévoile d'office, quoiqu'inconsciemment, la conclusion qu'imposent ces récits : qu'il n'est pas de fait s'il n'est dit, qu'il n'est pas de vérité de la découverte de l'autre qui ne soit médiatisée par une perception, une subjectivité, une culture et un langage ; et que l'expérience du voyage ne se comprend, et même ne se construit, que par le mot. Il n'y a pas de monde, pas de pur «hors-texte», mais des visions du monde qui sont toujours-déjà «textes». Cela d'ailleurs est avoué, à l'incipit du voyage suivant, Twelve Days ${ }^{5}$ :

For a long time I believed that it would be impossible to make a book out of these experiences; I could see no shape in them, no pleasing curve; nothing but a series of anti-climaxes, and too much repetition of what I had done, and written down, before. Yet I was loath to let the whole thing go unrecorded. Was it for this that I had gone footsore, cold, hot, wet, hungry? Climbed up, and scrambled down? Covered all those miles? looked at all those goats? Surely not. There must be a book in it somewhere. The book was always in my mind, teasing at me, and little by little as time receded, it began to take shape, a meaning began to rise up out of the welter, a few definite conclusions which really had some bearing on halfformulated ideas. Besides, the fingers that have once grown accustomed to a pen soon itch to hold one again: it is necessary to write, if the days are not to slip emptily by. (TD 9)

"There must be a book in it somewhere $"^{6}:$ cette phrase est une confession. Car le modal pose une proposition qui est de l'ordre de la spéculation, mais aussi et surtout, derrière cette façade hypothétique, de l'ordre de l'impératif catégorique, pour l'écrivain qui ne se résout pas à se défaire de son parti-pris de «littérariser » le monde, de transformer le monde en texte. Ce modal est d'ailleurs un symptôme, car il ressurgit dans les lettres de Vita Sackville-West à Virginia Woolf. Arrivée à Delhi le 14 février 1926, lors son trajet vers Téhéran, Vita écrit à son amante : «I must write about all these things, and naturally my stock is not infinite though rich. In fact I must write a traveller's tale, when I get to Persia and have a writing table instead of a knee to write on. I was never at my best on my knee $»^{7}$. Il faut donc écrire le voyage, pas seulement pour le communiquer, mais avant tout pour lui donner forme, en ordonnançant les faits et en sélectionnant pour créer le 
relief dans une série continue et désordonnée de non-événements ("anti-climaxes", disait-elle dans Twelve Days). Seul l'acte de narration fera jaillir la signification en structurant une réalité qui fût sinon restée brute et informe : «it began to take shape, a meaning began to emerge out of the welter ». C'est dire encore l'impossibilité de faire sens, d'interpréter le voyage dans l'immédiateté de l'expérience; et donc l'impérieuse nécessité de changer cette matière insignifiante en texte, sur le champ. Cette dernière expression est d'ailleurs à saisir littéralement, puisque c'est au cours du voyage (« on the spot », pour rendre l'expression «sur le champ » dans son sens spatial) que s'élabore le récit. Le 23 février, avant même d'avoir atteint la Perse, Vita écrit à Virginia : « And now I am quite spry again, and have even written six pages of my new book» (Letters 106). Le processus d'écriture est d'ailleurs mis en abyme dans le récit lui-même, au beau milieu de la traversée maritime d'Inde vers l'Irak: "I fetched a deck-chair, pen and paper and began this book » $(P T 56)^{8}$. Du milieu de l'océan indien, elle avait écrit de même à Virginia Wolf : « But by the time I come home I shall have written a book, which I hope will purge me of my travel-congestion, even if it serves no other purpose » (Letters 99). L'écriture est donc là pour "purifier» le voyageur de son voyage - fonction thaumaturge, presque sacrée : traverser, transcender le simple fait pour atteindre à l'écriture. Car hors de cette nécessité de soumettre les faits à une forme, l'écriture est aussi compulsion irrésistible qui fourmille au bout des doigts : « the fingers which have once grow accustomed to a pen soon itch to hold one again. It is necessary to write». Vidé de ses motivations intrinsèques, le voyage ne sert-il finalement d'autre but que de s'acheminer vers l'écriture, que d'écrire - écrire non pas transitivement (écrire le voyage), mais intransitivement: écrire tout court, écrire pour écrire ? "Travel is simply a text " pourrait-on dire en paraphrasant l'introduction de Passenger to Teheran dans laquelle Vita Sackville-West avait affirmé : « Travel is simply a taste » (29).

C'est l'objet ici : montrer que le récit n'est pas seulement le point d'aboutissement du voyage, mais y est toujours-déjà présent. Le voyage est comme un hors-texte autour duquel se tisse tout un feuilleté d'écritures plurielles, à lire comme autant d'exercices de style. Autour de l'expérience persane rayonnent en effet au moins trois niveaux d'écriture et de réécriture, ou de transposition, depuis l'investissement le plus personnel jusqu'au texte le plus distancé de son énonciatrice; depuis l'exclamation spontanée jusqu'à la rhétorique et ses figures. En premier lieu viennent les lettres envoyées à Virginia Woolf : jaillissement premier, expression de l'émotion à partager, en l'absence encore de toute formalisation (ce qui ne signifie pas que l'écriture n'y soit pas consciente d'elle-même, bien au contraire) ; puis les deux récits de voyage publiés en 1926 et 1928, qui reviennent sur les traces des lettres, mais leur imposent cette "mise en forme" voulue par Vita; enfin, ses poèmes, notamment les deux grandes pièces narratives The Land et The Garden, où viennent irradier, non plus les faits du voyage, mais les images glanées en chemin, devenues métaphores et tropes poétiques. En fait, les trois niveaux ne sont pas chronologiquement organisés mais contemporains les uns des autres, s'entrelaçant et s'interpénétrant; et les échanges ne s'y font pas à sens unique, de la correspondance vers les récits puis la poésie, mais en multiples transpositions ou traversées croisées. Ainsi la phrase d'introduction de Passenger to Teheran, «Travel is the most private of pleasures » (25) est-elle répétée textuellement à Virginia Woolf dans une lettre envoyée lors du second voyage en Perse (Letters 172) ${ }^{9}$. De même, le poème The Land commencé avant le départ en Perse fut corrigé et enrichi d'images persanes tout au long du voyage, ainsi qu'au retour. C'est cette circulation vitale de tous les textes qui se 
complètent et se fécondent, cette ronde de l'écriture autour de l'expérience centrale du voyage, que je voudrais étudier, pour montrer comment l'image « exotique » se constitue peu à peu dans l'affranchissement progressif du contexte, et le passage au texte poétique : ces images en effet jaillissent spontanément dans la correspondance pour ressurgir ensuite dans les récits de voyage, mais ne prennent finalement toute leur signification, tout leur relief, qu'une fois sorties de leur contexte premier, déplacées pour venir faire saillance dans la poésie pastorale de Vita Sackville-West.

\section{La correspondance avec Virginia Woolf}

6 Lorsque Vita Sackville-West se voit contrainte d'embarquer pour la Perse le 20 janvier 1926, elle vient tout juste de plonger dans une idylle passionnée avec Virginia Woolf, et leurs lettres sont traversées de soupirs amoureux qui vont de la simple ponctuation émotionnelle ("I just miss you, in a quite simple desperate human way ", Letters 89) à des lamentations étendues parfois à des paragraphes entiers :

Like a little warm coal in my heart burns your saying that you miss me. I miss you oh so much. How much, you'll never believe or know. At every moment of the day. It is painful but also rather pleasant if you know what I mean. I mean, that it is good to have so keen and persistent a feeling about somebody. It is a sign of vitality (no pun intended). (Letters 110)

Ce déchirement affleure dans l'introduction de Passenger to Teheran, et menace même de lui faire rater son objet puisque, de façon révélatrice, il lance le propos non pas sur la question du récit de voyage, mais sur l'incapacité de l'écriture épistolaire à communiquer une expérience. En effet, la lecture de la lettre, argumente Vita Sackville-West, est toujours décalée dans le temps par rapport aux faits rapportés; son destinataire ne pourra donc jamais communier dans l'événement avec l'auteur de la missive. En contrepartie, ce décalage place aussi la lettre dans le temps de l'attente et donc du désir, qu'elle entretient et aiguise. Plus grave est l'autre faille de la lettre, à savoir que le référent, le paysage décrit, n'y a pas le même degré de réalité pour l'auteur et pour son lecteur - d'un côté une expérience vécue, de l'autre un monde tout au plus reconstitué en imagination. Le débat, en cette introduction, est donc subrepticement déplacé du récit à l'échange épistolaire, et l'on devine pourquoi : Vita a tellement attendu et désiré de lettres $^{10}$ que son trajet est encore empreint rétrospectivement de cette tension douloureuse. Mais réfléchir à l'écriture du récit par le biais de la correspondance, c'est confondre description et communication, ou pour reprendre les catégories de Jakobson, confondre la fonction référentielle (celle dominant le récit, sans toutefois y agir seule) et la fonction émotive, à la fois expressive (du côté du destinateur) et conative (du côté du destinataire). Les lettres ne décrivent pas, ne tentent pas même de donner à voir, d'esquisser l'image d'un monde. Elles représentent le stade «vif » de l'écriture, où la description est tout simplement empêchée par le débordement d'émotion. Ainsi sur le bateau Rajputana, en route vers l'Inde : «Too many emotions, and too much Egypt, and too much excitement » (Letters 95). Et lorsque la lettre tente de « donner à voir », c'est sur un mode condensé et, dirait-on, " précipité », tel un cristallisé d'expérience :

Now I shall not tell you about Persia, and nothing of its space, colour and beauty, which you must take for granted [...] You smile? Well, I have stuck in a river, crawled between ramparts of snow, been attacked by a bandit, been baked and frozen alternately, travelled alone with ten men (all strangers), slept in odd places, eaten wayside meals, crossed high passes, seen Kurds and Medes in caravans, and 
running streams, and black lambs skipping under blossom, seen hills of porphyry ${ }^{11}$ stained with copper sulphate, snow-mountains in a great circle, endless plains, with flocks on the slopes. Dead camels pecked by vultures, a dying donkey, a dying man. Come to mud towns at nightfall, stayed with odd gruff Scotchmen, drunk Persian wine. Worn a silk dress one day, and a sheepskin and a furcap the next [...] But all this, as you say, gives no idea at all. How is it that one can never communicate? Only imaginary things can be communicated. But not real experience. (Letters $111-112)^{12}$

Le passage est le noyau, la condensation d'intensités d'où semble avoir jailli par la suite tout le texte de Passenger to Teheran, et l'on pourrait reconnaître en chacune des allusions ponctuelles de la lettre un des épisodes développés extensivement dans le récit de voyage. C'est d'ailleurs souvent le cas au fil des lettres, et certaines des anecdotes contées à Virginia, comme l'histoire pathétique et comique de ce voyageur européen dépouillé de toutes ses possessions par des bandits de grand chemin et contraint d'entrer dans Chirâz vêtu de sa seule veste à queue de pie, portée sens devant derrière, voyagent presque telles quelles de la correspondance (Letters 182) vers le récit de Twelve Days. La correspondance est donc le petit laboratoire itinérant de l'œuvre narrative à venir - et c'est là un rapport usuel entre les deux formes d'écriture. Mais, comme semble s'en désespérer Vita Sackville-West, les deux formes ne "communiquent " pas de la même manière (c'est d'ailleurs pourquoi elle a tort de désespérer...). Car les lettres ne communiquent certes pas un contenu, mais vibrent d'un rythme qui restitue l'émotion, la perception singulière de l'expérience, faite ici d'étonnement amusé, et rendue par toutes les syncopes de l'écriture : d'interminables énumérations, souvent nominales, où la paraphrase juxtapose les expériences les plus incongrues, au risque de la rupture de construction, et où le rebond constant par-delà la virgule rend sensible le tempo effréné d'une phrase qui peine à rendre compte de la diversité vertigineuse des scènes vues. Cette écriture « à vif » tend toujours vers l'énumération, l'exclamation, l'interjection, et trouve son aboutissement logique dans le cri passionnel: «Lord, what a funny place to be in - and those wild unknown mountains lying about - and night coming out - and the sound of the muleteers moving about - and a little snatch of song. Oh if only you were here! I am alone with four men. » (Letters 192). C'est peut-être ce "devenir cri » de la lettre (cri de surprise, cri d'incrédulité devant les richesses du pays offertes en spectacle, mais aussi cri du désir et cri d'amour) qui lie le référentiel et l'émotif, et les porte tous deux sur le rythme de l'adresse haletante. D'où le sentiment que les lettres si spontanées sont en fait plus suggestives, plus «visuelles » et plus intenses, dans leur fragmentation même, que les récits de voyage soigneusement recomposés autour de descriptions attentives. Ainsi d'une visite au Shâh qui, esquissée sur ce ton lapidaire de la correspondance, semble tout droit sortie des Mille et une nuits :

Just back from the palace, with half an hour before the bag shuts ${ }^{13}$

I am blind. Blinded by diamonds.

I've been in Aladdin's cave.

Sacks of emeralds were emptied out before our eyes. Sacks

of pearls. Literally.

We came away shaking the pearls from our shoes. Ropes of

uncut emeralds. Scabbards encrusted with precious stones.

Great hieratic crowns.

All this in a squalid room, with grubby Persians drinking little

cups of tea.

I can't write about it now. It was simply the Arabian nights, 
with décor by the Sitwells. Pure fantasy. Oh, why weren't

you here? (Letters 120) ${ }^{14}$

L'épisode, longuement retravaillé dans Passenger to Teheran, ne peut qu'y perdre l'expression scandée de la stupeur : y subsistent les références (à Aladin, par exemple), le vocabulaire employé (avec des mots comme « hieratic»), certaines vignettes (comme les Persans buvant leurs « petites » tasses de thé), pourtant la spontanéité est perdue - sans doute parce que l'immédiateté de la perception rendue dans la lettre par toutes les ellipses du style (parataxe, phrases nominales, répétitions) y est remplacée par des présentatifs («became ", " displaying», "came again») et des modalités appréciatives ( « enormous », « barbaric ») qui diluent la force du merveilleux en confisquant au lecteur une partie de sa surprise :

Suddenly, and as with a physical start, my eyes and thoughts came together, as gears engaging: I stared, I gasped, the small room vanished; I was Sinbad in the Valley of Gems, Aladdin in the Cave. The linen bags vomited emeralds and pearls; the green baize vanished, the table became a sea of precious stones. The leather cases opened, displaying jewelled scimitars, daggers encrusted with rubies, buckles carved from a single emerald, ropes of enormous pearls. Then from the inner room came the file of servants again, carrying uniforms sewn with diamonds; a cap with a tall aigrette, secured by a diamond larger than the Kuh-i-Nur; two crowns like great hieratic tiaras, barbaric diadems, composed of pearls of the finest orient. (PT 129)

Alors que la correspondance est nichée au creux de la subjectivité, et placée au plus près de la sensation, notamment par le biais de l'exclamation, les deux récits de voyage rendent donc apparent l'effort de "mise en texte» - voire de mise en scène... La subjectivité n'y subsiste que tempérée, sous la forme d'un humour très britannique se tenant à une distance amusée des scènes vécues ${ }^{15}$. Il semble qu'il faille calmer l'ardeur de l'écriture pour « faire texte».

\section{Passenger to Teheran et Twelve Days : récits du voyage}

D'emblée pourtant le voyage semblait se dérouler à travers des textes autant qu'à travers des paysages: le premier voyage se fait accompagné des œuvres complètes de Shakespeare, et comme si cela ne suffisait pas, de celles de Proust (auxquelles s'ajoutent Boswell, De Quincey, Fielding, Plutarque, etc., Letters 183)! Nulle surprise dès lors si un long développement inaugural est consacré aux agréments et désagréments des lourdes malles, valises et bagages... Mais la présence de ces deux œuvres-mondes est aussi révélatrice: car, s'extasie la narratrice, la force de Proust à construire un univers cohérent et autonome n'est nulle part aussi frappante que dans un contexte radicalement autre, c'est-à-dire vu depuis les frontières d'un autre monde (PT 57). Ainsi la littérature se voit-elle revivifiée par le voyage, tout comme le voyage est lui-même déterminé par une longue filiation de textes littéraires. Dans Twelve Days, les préparatifs au départ consistent en lectures de cartes et en consultations comparatives de sources érudites longuement citées - et de manière assez inattendue pour un récit de voyage, le texte s'achève sur une bibliographie d'une dizaine de titres, où figurent tous les grands explorateurs et archéologues victoriens de la Perse: Sir Henry Rawlinson, Sir Henry Layard, Lord Curzon... Le pompeux sous-titre à rallonge dont est assorti Twelve Days (« An Account of $a$ Journey in the Bakhtiari Mountains of South-Western Persia ») trahit lui aussi cette prétention encyclopédique inattendue. Mais c'est que le voyage le plus fascinant s'effectue sur les traces d'autres voyageurs illustres, et les grandes épopées exploratoires restent toujours à 
l'horizon: Vita Sackville-West se réjouit d'entrer en Perse par le chemin terrestre de la frontière irakienne, suivi certes par Alexandre, mais jamais foulé par Marco Polo! C'est donc à travers la littérature qu'a d'abord retenti l'appel de la Perse : celle des jardins d'Éden, de Théocrite et de la Bible (TD 69), mais aussi celle des poètes (Herbert ou Hâfiz, TD 24) ; celle, enfin, de Conrad dont le spectre, omniprésent, permet de caractériser toutes les scènes déroutantes où se révèle un ailleurs plus étrange qu'exotique. Les noms de lieux sont chargés d'avance de la poésie des siècles passés. Aussi le regard est-il toujours un regard "prévenu ", informé, aux deux sens du terme : regard savant, mais aussi modelé, dirigé, déformé par ce savoir littéraire. Une longue étude de type postcolonial serait à donner de ce regard "qui sait ", ou présume savoir, avant même d'avoir vu les scènes elles-mêmes. Il serait assez aisé de traquer au fil des textes de Vita Sackville-West les manifestations de cet « œil impérial », pour reprendre l'expression de la critique Mary-Louise Pratt. Même si le second voyage se fait au pays des Bakhtiari, les contacts avec la population sont pratiquement inexistants et en tous cas superficiels ${ }^{16}$ (TD 62); et le grand voyage intrépide s'achève finalement emprisonné dans le petit monde (effectivement très proustien) des diplomates, européens expatriés et aristocrates voyageurs - autre réminiscence des lectures de la voyageuse ${ }^{17}$ (Letters 180). Les généralisations de l'auteur, et sa manière presque constante de «typifier » le Persan, l'hypocrisie presque spontanée des habitants, leur "apathie» naturelle (TD 92), l'« ignorance » du peuple ${ }^{18}$ (TD 79), les goûts « dégénérés », et la naïveté des Orientaux en

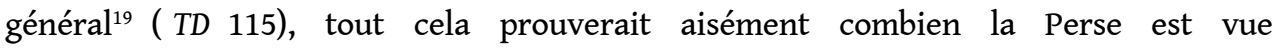
(inconsciemment sans doute) au prisme d'une idéologie impérialiste. Cette position de tranquille domination est d'ailleurs plus insistante dans le second voyage, où le sens de la découverte émerveillée semble s'être émoussé et où la voyageuse, déjà assurée d'être en terrain connu, se laisse aller à trancher catégoriquement sur ce qu'est l'âme persane, toujours en quête de cette essence de l'être dont elle sent pourtant confusément le caractère élusif.

12 À travers la correspondance avec Virginia Woolf, on saisit pourtant qu'au-delà de ce prisme culturel, il est un autre prisme déformant apposé sur l'aventure persane : celui de la rivalité littéraire. L'amour passionné ressenti par Vita pour Virginia jaillit en effet du désir de l'aventurière aristocrate et rebelle de protéger et même de materner un être qu'elle sait éminemment fragile, désir auquel se mêle une admiration éperdue pour l'infini talent de la romancière. Au moment où Vita quitta l'Angleterre, Virginia Woolf venait de publier Mrs Dalloway, et ses lettres faisaient partager à son amante l'excitation fébrile dans laquelle la plongeait la rédaction de To the Lighthouse. Or, dans la correspondance, l'admiration de Vita pour Virginia ne va jamais sans le sentiment douloureux de ses propres limites, et les compliments à l'aimée sonnent immanquablement comme des auto-flagellations: «I have just lunched [...] with Mrs Dalloways [sic] open on my knee and am consequently in a mood of intellectual humility $»^{20}$ (Letters 84). Ailleurs, elle parle de la romancière à la troisième personne du singulier, comme par révérence : «I don't know whether to be encouraged or dejected when I read the works of Virginia Woolf » (Letters 105). Et au milieu de son travail sur Passenger to Teheran: "[I] have written six pages of my new book. It is a rambling, discursive sort of affair. And I think of your lovely books, and despair » (Letters 106); "I've written a good deal but all pretty bad (I send a chunk to Leonard by this post), and envy your thousands of words » (Letters 122). Le fait que les deux récits de voyage aient initialement été publiés, et donc inévitablement lus et jugés, par Leonard Woolf ne faisait 
sans doute qu'attiser le complexe littéraire de Vita. Aussi après une réponse encourageante de Leonard, elle disait son soulagement : " I am relieved to find that I am not quite an idiot after all» (Letters 140). Et après une lecture et une appréciation (pourtant fort mesurée) de ses poèmes par Virginia : «I'm glad you thought The Land looked pretty ${ }^{21}$ (Letters 143). La relation esquissée par la correspondance étonne par ce complexe mélange d'amour et de sourde rivalité littéraire. Et l'on se demande à cette lecture si Vita Sackville-West n'a pas choisi le genre du récit de voyage parce qu'il lui imposait d'adopter la position inverse à celle de l'amante géniale - ou plus exactement une position opposée et complémentaire tout à la fois. L'une, Virginia, sonderait et se perdrait dans des intériorités ; l'autre, Vita, serait contrainte par la nature et la distance même de son sujet de se poser en parfaite extériorité par rapport aux choses vues. Deux explorations distinctes donc, l'une de paysages intérieurs, l'autre de décors étrangers. «I was determined to go through Persia with an eye to outward appearances only " écrit Vita dans Passenger to Teheran (PT 104).

13 Cependant son récit ne cesse de trahir cet objectif avoué. Car, sent-elle à de multiples instances, le voyageur amène son regard, qui n'est pas seulement informé par une culture et une littérature : il est aussi intensément personnel et subjectif. À l'orée de Passenger to Teheran, une citation de Kinglake, autre voyageur littéraire, lui permet de poser une définition du point de vue que ne récuseraient pas les narratologues modernes travaillant sur la focalisation interne : « [the traveller's] very selfishness - his habit of referring the whole external world to his own sensations, compels him, as it were, in his writings, to observe the laws of perspective - he tells you of objects, not as he knows them to be, but as they seemed to him » (PT 30). De même, Vita se défend vigoureusement d'offrir un livre instructif, fondé sur des données objectives : « Learned I am not; well-read, only in scraps; polemical not at all; didactic: I hope not, but am not very sure » (PT 31). Si Twelve Days abonde en déclarations de savoir et de pouvoir impérialistes, le récit précédent en revanche insiste systématiquement sur la relativité et les limitations des points de vue individuels : « Different persons observe different things » (PT 66-67). Et, s'extasiant sur la couleur locale des bazars de Téhéran, la narratrice en vient à imaginer la même scène, forcément déplacée, forcément grotesque et dévaluée, si l'objet contemplé était tout simplement le marché de Smithfield (PT 94) : la perception de l'étrangeté est elle aussi question de point de vue. En retour cependant, les scènes contemplées obligent le spectateur à repositionner tout son schéma perceptif et à reconsidérer ses idées anciennes à partir d'une situation légèrement décalée :

This question of horizon, however; how important it is; how it alters the shape of the mind; how it expresses, essentially, one's ultimate sense of country! That is what can never be told in words: the exact size, proportion, contour; the new standard to which the mind must adapt itself. (PT 69)

Cette constatation de la relativité des points de vue dans l'espace et le temps est la réponse, contenue dans le texte lui-même, aux déplorations initiales sur l'impossibilité à toucher jamais à la "réalité » du monde : il n'y a tout simplement pas de réalité du monde, il n'y a que des perceptions et des reconstructions individuelles du monde. Et c'est cette intuition qui fait de Vita Sackville-West une véritable " édouardienne ", selon le titre de l'un de ses romans les plus connus, placée entre l'assurance satisfaite qu'avaient les Victoriens de dominer le monde, et l'inconfortable prise de conscience de la relativité culturelle et idéologique des points de vue - conscience encore floue, mais anticipant étrangement sur les positions postcoloniales. C'est cette ambiguité qui fait vaciller l'adjectif possessif dans l'exclamation finale, « Where was my Persia?» (PT 146). 
Ce possessif peut s'entendre en deux sens au moins : comme allusion autoritaire à un territoire qui lui appartienne en propre - c'est là la lecture impérialiste et « victorienne ». $\mathrm{Ou}$, ce qui est bien différent, comme référence affectueuse à un territoire qu'elle seule sache percevoir, percer à cœur, et réécrire - selon une lecture résolument moderne, anticipant la critique postcoloniale.

\section{The Land et The Garden : la poésie, fruit du voyage}

Il est dans la correspondance à Virginia Woolf une petite vignette humoristique, un dessin à main levé, où s'illustre la diversité de la carrière littéraire de Vita SackvilleWest : «I was at the crossways just about the time I met you » confie-t-elle à Virginia ${ }^{22}$ ( Letters 166). Le dessin représente une borne de signalisation pointant dans deux directions opposées, l'une indiquant vers la gauche "bad novels ", et l'autre fléchant vers la droite "good poetry ». Manière de dire l'avenir de l'écriture, c'est la poésie; ou plus exactement (puisque les deux pratiques s'entremêlent et que la relation entre prose et poésie n'est pas de consécution dans l'œuvre de Vita Sackville-West) que la réalisation suprême de l'écriture, c'est la poésie ; et que l'exemple et la présence de Virginia Woolf furent capitaux dans ce chemin de l'écriture vers l'excellence. Vita avait emmené en Perse l'ébauche de son poème The Land, commencé à la fin de l'été 1925, et qui devait être publié peu après son retour en Angleterre, et couronné par le Hawthornden Prize. Elle l'étoffa durant son voyage et l'enrichit d'images persanes, ainsi que d'autres brefs ajouts, dont certains composés sur place. C'est le cas d'un poème envoyé directement de Téhéran à Leonard Woolf, pour publication : «I have finished my poem [...]. It goes off by this bag. ${ }^{23}$ There are large patches of Persia in it now. Will you approve, I wonder? ${ }^{24}$ (Letters 118). Elle écrit également au sujet des ajouts apportés à The Land: "I have added bits to my poem, but they are nothing without context. As you may suppose, they are almost entirely Asiatic in character ${ }^{25}$ (Letters 117). "Nothing without context» dit-elle. En se trompant. Car c'est précisément lorsque les images persanes sont extraites du récit où leur valeur était essentiellement référentielle, pour être insérées dans la poésie, qu'elles y prennent un relief nouveau, celui de la métaphore, fondée par définition sur ce transport de sens. Bien loin de tuer l'image, c'est cette opération d'extraction et de décontextualisation qui fait d'elle la touche exotique au sein du familier.

poésie de Vita Sackville-West ${ }^{26}$ exploite largement la capacité du nom étranger à ouvrir tout un imaginaire, capacité qu'elle avait déjà souligné dans Twelve Days (81) : « To me, remote places hold the magic touch the romantic names held for Marlowe and Milton. (It is my only justification for writing books of travel)» :

I have known bees within the ruined arch of

Akbar's crimson city

[...] the Great Moghul lolling on his throne

Between his languid fingers crumbling spice

(from The Land, Poems 49)

Le bref suspens créé à la rime prolonge et renvoie les échos du nom exotique :

Shall we go back, my flute and I

To find again the shepherd of Shalil,

And when the planet Venus travels high

Above the myrtle grove of Murdafil,

Make each our separate music? For I too 
Am silent sometimes, thinking of those stars,

That solitude, those evenings waning blue,

And the lost beauty of the plains of Fars.

(« The Persian Flute », Poems 174-175)

Mais plus souvent, l'image prend son relief d'un rapprochement inattendu: la ressemblance formelle et visuelle, par exemple, entre un ciel anglais ténébreux et une tente de nomades: "But now as black as any nomad's tent, the night-time and the night of time have blent / their darkness» («Sissinghurst $»^{27}$, Poems 114). Au milieu du «Printemps », l'une des quatre saisons qui organisent le cycle de The Land ${ }^{28}$, surgit l'un de ces moments de radicale défamiliarisation, sur le ton nostalgique d'un « Ubi sunt »... Mais le déplacement est spatial tout autant que temporel :

I have known honey from the Syrian hills

Stored in cool jars; the wild acacia there

On the rough terrace where the locust shrills,

Tosses her spindrift to the ringing air;

Narcissus bares his nectarous perianth

In white and golden tabard to the sun

And while the workers rob the amaranth

Or scarlet windflower low among the stone

Intent upon their crops,

The Syrian queens mate in the high hot day,

Rapt visionaries of creative fray.

(The Land : " Winter »)

Le seul terme "Syrian» fait basculer dans un autre monde ce qui aurait pu n'être que l'image convenue d'une terre promise de douceur et de miel. Un instant, les collines et les terrasses plantées d'acacias et écrasées sous la chaleur d'un jour torride (" high hot day ») font croire à une scène « exotique ». Et pourtant l'ailleurs dont il est question ici n'est pas un lieu ou un territoire qui se donnerait à la description. C'est un composé de scènes et de sons, où l'effet de défamiliarisation jaillit à la fois des fragments de choses vues et remémorées ( " patches of Persia », disait-elle), et de l'étrangeté sonore de ces « nectarous Narcisses ", " perianth », " amaranth », et autres fleurs méconnues de la botanique et de la phonétique.

Vita Sackville-West se plaisait à parler à Virginia Woolf de ses « Géorgiques » (Letters 69), et sa poésie est tout emplie de sa passion horticole. Le retour de Perse marque à cet égard comme une seconde carrière. À la mort de son père, Vita perdit en effet le château de Knole, qui durant des siècles avait appartenu à sa famille, mais fut alors transmis à un neveu, seul descendant masculin. Pour tenter de compenser cette "perte monumentale", pour reprendre le jeu de mots de sa biographe ${ }^{29}$, Vita et son mari Harold Nicolson firent l'acquisition, en mars 1930, des ruines du château de Sissinghurst, qu'ils entreprirent de relever. La « baroudeuse » qu'elle avait été s'enferma presque en recluse, et se consacra à la création d'un jardin aujourd'hui encore connu de tous les amateurs. C'est aussi cette passion pour la botanique et l'horticulture qui lui fit publier articles et ouvrages, à un rythme soutenu et avec un souci presque encyclopédique de complétude et de systématicité : Country Notes (1939), In your Garden (1951), In your Garden again (1953), More for your Garden (1955), Even More for your Garden (1958), Vita Sackville-West's Garden Book (1968)... Ce sont ces deux inspirations, l'exotique et l'horticole, qui se croisent sans cesse dans ses poèmes, où l'expérience du voyage ne semble servir qu'à ramener par défaut à l'amour de la terre et de l'enracinement dans le sol natal. 
21 À rebours, ces deux veines indémêlables agissent par rebond sur les récits de voyage, où elles donnent une nouvelle saillance à des notations ou des images qui seraient restées inaperçues, faute de tisser un réseau figural assez dense. Apparaissent alors nouvellement la minutie et la précision mises à repérer, identifier, et différencier les plantes au bord du chemin : jacinthes, tulipes sauvages, jonquilles, renoncules, asphodèles, coquelicots... Ainsi, la lecture et la connaissance de la poésie de Vita Sackville-West transmuent en retour le ton des récits de voyage, que l'on pourrait lire d'un point de vue nouveau, comme quête de la plante mythique qu'est le fameux iris persan (" iris reticulata »), cet iris qui n'est pas bleu mais pourpre et or (TD 49 sq., PT 90-91)... Le mouvement se fait donc d'abord du hors-texte vers le texte, faisant glisser les images nées du voyage vers le "travelogue", puis la poésie; mais aussi, dans une série de transpositions plus complexes, d'un texte à l'autre, réinvestissant les métaphores florales qui émaillent la poésie bucolique de Sackville-West au sein de l'épopée persane, en un tourbillonnement constant de l'image vue à l'image rêvée et recomposée, en mots et en purs sons. Finalement, le voyage de découverte n'était sans doute qu'un voyage au plus profond de la passion personnelle, qu'une première plongée au cœur de l'écriture et de ses pouvoirs de transmutation de l'espace, ainsi que Vita l'avait promis à Virginia avant son départ ( Letters 72) : «I shall make the desert flower ${ }^{30}$.

L'écriture fait «fleurir » le lieu parce qu'elle ne se contente pas de faire un récit ponctuel du voyage, mais l'ouvre en images diverses, qui vont éclore et disséminer dans l'œuvre tout entière. Ce que fait le voyage dans l'œuvre, c'est donc d'assurer une transition vitale entre les différents moments d'une écriture, une circulation d'énergie qui ne porte pas seulement le lecteur du récit d'exploration vers les poèmes, mais reflue aussi des métaphores poétiques vers la prose, pour y donner saillance et cohérence à l'inspiration "géorgique ». C'est ce rayonnement interne à l'œuvre, infiltrant l'exotique au sein du familier et le familier au sein de l'exotique, qui permet aux images que l'on eût cru platement « horticoles » de se révéler en fait bien plus que cela : l'idiome poétique de Vita Sackville-West peu à peu constitué par la cristallisation des images et des sons. C'est dans ces instants de défamiliarisation, par ces brusques échappées hors du référent, par ces transpositions de l'expérience voyageuse en images voyageuses, que peut se découvrir la voix du texte, le poème de l'ailleurs.

\section{BIBLIOGRAPHIE}

De Salvo, Louise, \& Mitchell A. Leaska. The Letters of Vita Sackville-West to Virginia Woolf, New York: William Morrow and Company, 1985.

Sackville-West, Vita. Passenger to Teheran. New York: Harper Perennial, 1990 ( $1^{\text {st }}$ published, Hogarth Press, 1926). Trad. Une Anglaise en Orient. Paris : 10/18, 1993.

Sackville-West, Vita. Twelve Days: An Account of a Journey in the Bakhtiari Mountains of South-western Persia, London: Michael Haag ltd, 1987 ( $1^{\text {st }}$ published, Hogarth Press, 1928). Trad. Une Aristocrate en Asie. Paris : Anatolia, éditions du rocher, 2000. 
Sackville-West, Vita. Collected Poems, New York: Doubleday, 1934.

\section{NOTES}

1. Claude Lévi-Strauss, Tristes Tropiques, Paris : Plon, coll. Terre Humaine, 1955.

2. Vita Sackville-West, Passenger to Teheran, New York: Harper Perennial, 1990 ( $1^{\text {st }}$ published, Hogarth Press, 1926), 25. Désigné ci-après comme PT.

3. Olivier Hambursin, «Les préfaces des récits de voyage du XX $\mathrm{X}^{\mathrm{e}}$ siècle : expression de l'ambiguïté et de la spécificité d'un genre? ", Seuils et traverses : enjeux de l'écriture du voyage (vol. 2), Jan Borm (éd.), Brest, 2002, 283.

4. Ibid., 284.

5. Twelve Days: An Account of a Journey in the Bakhtiari Mountains of South-western Persia, London: Michael Haag ltd, 1987 (1 $1^{\text {st }}$ published, Hogarth Press, 1928). Désigné ci-après comme TD.

6. C'est moi qui souligne.

7. Louise De Salvo \& Mitchell A. Leaska, The Letters of Vita Sackville-West to Virginia Woolf, New York: William Morrow and Company, 1985, 102. C'est moi qui souligne. Ci-après Letters.

8. Vita rentra de Perse en mai 1926 et le récit fut publié par la Hogarth Press dès novembre 1926, ce qui prouve qu'il devait être déjà bien élaboré au retour du voyage, et qu'une large partie fut sans doute conçue dans le mouvement même du voyage.

9. Lettre du 9 février 1927.

10. L'acheminement de Téhéran à Londres pouvait prendre jusqu'à six semaines.

11. L'image des montagnes de porphyre revient à plusieurs reprises dans Passenger to Teheran, cf. par exemple 72 et 88 .

12. L'emphase est de Vita Sackville-West.

13. Il s'agit du « sac » postal.

14. L'emphase est dans le texte.

15. D'où peut-être le choix aussi désastreux que redondant des deux titres donnés aux récentes traductions françaises des récits : Une Anglaise en Orient (traduction de Passenger to Teheran, Paris : 10/18, 1993), et Une Aristocrate en Asie (traduction de Twelve Days, Paris : Anatolia, éditions du rocher, 2000.

16. « what were Arabs to me, or I to them?».

17. Lettre du 25 février 1927.

18. «It is not that these people are cruel, but they are ignorant ».

19. " the Orientals' delight in ingenuity ".

20. Lettre du 11 janvier 1926. Le titre du roman de V. Woolf n'est pas souligné dans le texte.

21. Lettre du 3 octobre 1926.

22. Lettre du 29 janvier 1927.

23. Allusion, à nouveau, au « sac » postal.

24. Lettre du 8 avril 1926.

25. Lettre du 15 mars 1926.

26. Voir Vita Sackville-West, Collected Poems, New York: Doubleday, 1934. Ci-après Poems.

27. Le poème est dédié à Virginia Woolf, et ouvre le recueil In England (1930).

28. Cette structure en saisons fut reprise dans The Garden, composé au moment du Blitz de Londres.

29. Letters, op. cit., introduction, 35.

30. Lettre du 13 octobre 1925 . 
INDEX

oeuvrecitee Garden (The), Land (The), Passenger to Teheran, Twelve Days: An Account of a Journey in the Bakhtiari Mountains of South-Western Persia 